\title{
Are Exotic Stable Quarks Cosmologically Allowed?
}

\author{
Enrico Nardi* and Esteban Roulet \\ International School for Advanced Studies, I-34014 Trieste, Italy \\ and \\ CERN - Geneva \\ * Also Istituto Nazionale di Fisica Nucleare, Sezione di Trieste, Italy \\ Ref.S.I.S.S.A. 38 EP (Mar 90)

\section{Abstract}

We examine the possibility that new exotic quarks, as for example the $Q=-1 / 3$ charged quarks predicted by $\mathrm{E}_{6}$ models, are stable. We show that their cosmological consequences combined with the bounds that arise from superheavy element searches, together with the bounds recently derived by requiring that heavy particles captured by neutron stars do not induce their collapse into a black hole, exclude that possibility. This implies that in these models some mechanism must exist to allow the exotic quark decay. 
Several GUT extensions of the standard model enlarge considerably the particle content of the three standard generations, and some of them include heavy 'exotic' quarks among the new particles. An interesting example are the $E_{6}$ models [1], like those arising from ten dimensional superstring theories after compactification to the four physical dimensions [2]. In these models each generation of fermions is assigned to a 27-dimensional (fundamental) representation that, together with the standard fifteen fermionic degrees of freedom, includes twelve additional new fields. The electromagnetic charge and colour quantum numbers of the new particles are univocally determined by the group structure. Besides the existence of new exotic charged and neutral leptons, each generation contains a new colour triplet and weak singlet quark $Q$ of electric charge $-1 / 3$. The mass of this quark, being model dependent, is in principle arbitrary, but a lower bound on it comes from present collider experiments. The LEP results exclude the existence of new quarks lighter than $\mathrm{M}_{Z} / 2$, while for hadron colliders this limit is $\sim 80 \mathrm{GeV}[3]$.

Since under the unbroken $S U(3) \times U(1)$ gauge group the heavy quarks transform with the same quantum numbers as the standard 'down type' quarks, a mixing among them is allowed. If this mixing is present, the $Q$ mass eigenstate should decay weakly into standard fermions. However, since the $S U(2) \times U_{Y}(1)$ quantum numbers are different, such a mixing induces deviations from the weak interactions of the down-type mass eigenstates predicted by the Standard Model and also flavor changing neutral currents. Under reasonable assumptions, present experimental data restrict the allowed value for the mixing angle to $\sin \theta_{m i x} \lesssim 0.02 / m_{Q}$ with $m_{Q}$ in $\mathrm{TeV}$ [4].

In this work we want to analyse the consequences of assuming the exotic quark to be stable (or nearly so, i.e. with lifetime larger than the age of the universe). For some superstring-inspired $E_{6}$ models, a stable exotic quark is a natural consequence of the particular structure of the superpotential. In fact in order to avoid low-energy baryon and lepton number violation, it is necessary to require that some potentially dangerous couplings vanish, and this is most easily done by introducing certain discrete symmetries. In turn, these symmetries often imply that the mixing between the exotic quarks and the ordinary down type quarks vanishes [4]. Although we have in mind the $E_{6}$ candidate, our analysis can be extended with minor modifications to other coloured particles (charge $2 / 3$ quarks, sextet quarks, ...).

If the heavy quark is stable, it can have important cosmological consequences. Its present density can be computed by following the thermal evolution of the universe. At very early stages its abundance is determined by the thermal and chemical equilibrium. 
Subsequently, the cooling of the universe reduces the annihilation rate of the heavy quarks until, at the freeze out temperature, the chemical equilibrium can no longer be mantained. However, as we will show the heavy particles remain in thermal equilibrium. In the confinement transition the exotic quarks hadronize together with the ordinary quarks. At this stage the relevant annihilation cross section associated with the disappearence of the heavy quarks increases and can reach a typical hadronic size, so that a significant reduction of the relic density of heavy hadrons takes place after confinement. (Our results on the present abundance of heavy quarks differ from a previous analysis that overestimated the annihilation cross section after confinement [5].) The superheavy hadrons are subject subsequently to primordial and eventually stellar nucleosynthesis, where heavy nuclei are also produced. The stringent experimental bounds from searches of superheavy elements are a powerfull test for the existence of these hypothetical stable exotic particles. We use also the bounds on very massive charged particles contributing to the cosmic dark matter that have been recently obtained from the study of their effects on the evolution of neutron stars [6]. These bounds, toghether with the cosmological requirements that the universe not be overclosed by these particles totally exclude the existence of a stable exotic quark.

In the following we assume that no particle-antiparticle asymmetry is present. This assumption does not affect the generality of our conclusions since an asymmetry can only increase the abundance of superheavies, giving more strength to the bounds obtained.

The equation describing the evolution of the number density $n$ of stable species is

$$
\frac{\mathrm{d} n}{\mathrm{~d} t}=-3 \frac{\dot{R}}{R} n-\langle\sigma v\rangle\left(n^{2}-n_{e q}^{2}\right)
$$

where $R$ is the scale factor of the universe, $\langle\sigma v\rangle$ is the thermally averaged annihilation cross section times the relative velocity and $n_{e q}$ is the value of the density in chemical equilibrium. The integration of this equation yields, for the relic mass density at present time $\rho_{o}[7]$

$$
\rho_{o} \simeq \frac{\sqrt{2 \pi}}{M_{P l}} s_{o}\left[\int_{x_{f o}}^{x_{o}} \frac{d x}{x^{2}} \sqrt{b}\langle\sigma v\rangle\right]^{-1}
$$

where $s=\frac{2 \pi^{2}}{45} g_{e f f} T^{3} \equiv(b T)^{3}$ is the entropy density, $g_{e f f}(T)$ is the effective number of degrees of freedom at temperature $T, x \equiv m / b T$ with $m$ the mass of the particle, and $M_{P l}$ the Planck mass. The freeze out value $x_{f o}$ is given by

$$
\left.x_{f o} \simeq \frac{1}{b}\left(\ln B-\frac{1}{2} \ln \frac{1}{b} \ln B\right)\right|_{f o}
$$


where

$$
B \equiv \Delta m \frac{M_{P l}}{2 \pi^{2} b^{2}}\langle\sigma v\rangle
$$

with the numerical factor $\Delta$, of order one, depending on the criterion used to define the freeze out temperature. (A fit to the numerical integration of eq. (1) leads to a preferred value $\Delta \simeq 1.5$.)

At temperatures above the confinement temperature $T_{c} \sim 200 \mathrm{MeV}$, the relevant annihilation cross section of two heavy quarks $Q$ involves the channels $Q \bar{Q} \rightarrow g g, q \bar{q}$, where $g$ is a gluon and $q$ an ordinary quark (we neglect the contribution to the annihilation cross section coming from electroweak channels). For a colour triplet quark in the nonrelativistic limit $(T \ll m)$ annihilating into $N_{f}$ lighter flavors, we obtain

$$
\langle\sigma v\rangle_{Q \bar{Q}}=\frac{\pi \alpha_{s}^{2}}{m^{2}}\left(\frac{2}{9} N_{f}+\frac{7}{27}\right) \text {. }
$$

In this expression, we take $\alpha_{s}\left(Q^{2}\right)$ renormalized to the scale $Q^{2} \sim m^{2}$, since this is the relevant momentum transfer involved in the annihilation. Assuming only the standard physics at energies below $m$, we get for instance $\alpha_{s}\left((10 \mathrm{TeV})^{2}\right) \simeq \alpha_{s}\left(M_{W}^{2}\right) / 2$. The appearence of new physics below the exotic quark mass could affect the value of $\alpha_{s}$ and could also open new channels for the annihilation. This is the case, for instance, if supersymmetry is present at the weak scale, since annihilations involving squarks and gluinos could contribute to eq. (5).

When confinement occurs, due to the presence of a relatively large number of ordinary quarks, the heavy quarks $Q$ will hadronize mainly forming a system of 'superheavy kaons' ( $Q \bar{q}$ and $\bar{Q} q$, with $q=u, d$ ) and, due to the baryon asymmetry, the $Q \bar{q}$ mesons will finish as superheavy baryons $Q q q$ through annihilations with ordinary nucleons.

In a previous study of the survival of heavy quarks [5], the annihilation cross section below $T_{c}$ was estimated to be equal to the ordinary nucleon-antinucleon cross section, $\sigma_{N \bar{N}} \sim 30 \mathrm{mb} / v$. However, several reasons indicate that this is an overestimate. In fact, in eq. (1) only the exclusive cross. section that does not contain the two heavy quarks in the final state should be used. Since we are considering energies below $\Lambda_{Q C D}$, the light quarks cannot be considered as spectators in the process of $Q \bar{Q}$ annihilation, which for instance could proceed through the hadronic process $\bar{Q} q+Q q q \rightarrow \bar{Q} Q+q q q$, with the formation of a $\bar{Q} Q$ bound state, which consequently decays into light particles. Although the associated cross section could be of hadronic size, since the Compton wavelength of $\mathrm{TeV}$ particles at $\mathrm{MeV}$ energies is $\lesssim \mathrm{fm}$, the total annihilation cross section cannot exceed the characteristic geometrical cross section associated to the range of the interactions $(\sim$ $\mathrm{fm}$ ), i.e. $\sigma \lesssim 4 \pi \mathrm{fm}^{2} \sim 100 \mathrm{mb}$. Instead, with the previously mentioned estimate of 
$\sigma \sim \sigma_{N \bar{N}}$ the very slow thermalized heavy hadrons (with $v=\sqrt{6 T / m}$ ) would have a cross section much larger than one barn. The heavy hadrons, unlike the ordinary nucleons, enter the long wavelength regime where the annihilation cross section can grow beyond the geometric one only at temperatures $(<\mathrm{MeV})$ where the densities have been too diluted for the annihilation to be efficient. Furthermore, the exchange of ordinary mesons $(\pi, \omega$, ...), that gives the main contribution to the total low energy annihilation of hadrons but does not affect the number of heavy quarks, should not be included in $\langle\sigma v\rangle$. Also, since the baryon asymmetry has washed out the ordinary antiquarks from the heavy hadrons, the vector exchange leads to repulsive interactions that further reduce the annihilation rate of $Q \bar{Q}$. In view of the previous discussion, we expect the relevant annihilation cross section to be much less than the corresponding geometrical cross section, and we will parametrize it as

$$
\sigma \equiv f \cdot 100 \mathrm{mb}
$$

with $f<1$.

In fig. 1 we show the resulting values of $\Omega h^{2}$ for the upper value $f=1$ and for $f=0.1$. Here $\Omega$ is the present relic mass density of heavy quarks in units of the critical closure density, $h$ is the Hubble constant in units of $100 \mathrm{~km} / \mathrm{s} \mathrm{Mpc}$ and, from observations, $0.4 \leq h \leq 1$. Due to the large uncertainty in the estimation of $f$ that is related with the non-perturbative effects involved, we have also plotted the other extreme case in which the annihilation rate after confinement is negligible. This would correspond to an annihilation process $\bar{Q} q+Q q q \rightarrow$ light hadrons proceeding essentially through a partonic-like cross section. In this case, $\Omega$ is determined with good accuracy ( $i n c e T_{f_{o}}>T_{c}$ ) by the free quark annihilation rate before hadronization. Quantitatively, it is safe to neglect annihilations below $T_{c}$ as long as

$$
\langle\sigma v\rangle_{T<T_{c}} \lesssim \frac{T_{f o}}{T_{c}}\langle\sigma v\rangle_{Q \bar{Q}} \sim \frac{m}{5 \mathrm{GeV}}\langle\sigma v\rangle_{Q \bar{Q}}
$$

where we have used a typical freeze out temperature $T_{\text {fo }} \sim m / 30-m / 20$.

Figure 1 clearly implies that masses larger than $\sim 10^{5} \mathrm{TeV}$ are cosmologically excluded, since they would yield $\Omega h^{2} \gtrsim 1$, overclosing the universe. Moreover, the observed lifetime of the universe suggests $\Omega h^{2} \lesssim 0.25$, making this constraint even stronger. For $E_{6}$ models, in the absence of any kind of mixing, each of the three flavours of exotic quarks contribute to $\Omega$, and then the cosmological bounds should be applied to the sum of their contributions. This leads to un upper limit of $m \sim 3 \cdot 10^{4} \mathrm{TeV}$ if the heavy quark masses are assumed to be similar. 
If the heavy quark is not a triplet of colour (sextets of heavy quarks have been considered e.g. in [5]), or if new physics is present at energies below $m$, the annihilation cross section would differ (but in principle not drastically) from the case previously discussed, and the value of the relic density $\Omega$, which is inversely proportional to it, will be correspondingly modified.

In the previous computation we have assumed that the heavy particles remain in thermal equilibrium. An argument to justify this assumption goes as follows: thermal equilibrium is mantained if the energy exchanged through collisions during an expansion time $\tau \simeq M_{P l} / T^{2}$ is larger than the original energy $E \sim T$ of the heavy particle, i.e.:

$$
n v \sigma \tau \Delta E \gtrsim E
$$

where $n$ is the number density of the scatterers, $v$ is their mean velocity, and $\sigma$ their typical cross section. Before confinement, thermalization proceeds mainly through scattering off termalized quarks and gluons through $t$-channel gluon exchange.' Although the corresponding cross section has a Coulomb-like divergence associated with the exchange of soft gluons in the forward scattering, the relevant quantity for the thermalization is the energy transfer cross section

$$
\sigma_{t r}=\int d \cos \theta \frac{d \sigma}{d \cos \theta}(1-\cos \theta)
$$

where $\theta$ is the center of mass scattering angle. Since inside the quark-gluon plasma the color charges undergo an 'electric' screening with a typical length [8] $m_{e l}^{-1}$ with $m_{e l}^{2} \simeq$ $(g T)^{2}\left(N+N_{f} / 2\right) / 3$ playing the role of an effective gluon mass $(N=3$ is the number of colors), after taking into account this effect we obtain a finite result:

$$
\left\langle\sigma_{t r}\right\rangle \sim \alpha^{2} \frac{m^{2}}{T^{4}} \ln \left(\frac{9 T^{2}}{m_{e l}^{2}}\right) .
$$

Since in this case the momentum transfer is $\Delta p \sim T, v \simeq 1$ and $n \sim T^{3}$, eq.(8) is always satisfied.

After confinement, taking into account only the scattering off nucleons, we can derive from eq.(8) an upper bound for the mass of a heavy hadron for it to be in thermal equilibrium: the baryonic asymmetry yields $n_{N} \sim 10^{-9} T^{3}$, while for non relativistic particles $v \sim \sqrt{T / m_{N}}$, and $\Delta E \sim T \sqrt{m_{N} / m}$, with $m_{N}$ the mass of the nucleon. We then obtain:

$$
m[\mathrm{GeV}] \lesssim 10^{12}(\sigma[\mathrm{mb}])^{2}\left(\frac{T}{\mathrm{MeV}}\right)^{3}
$$


We see that for a typical hadronic elastic cross section, the assumption of thermalization is correct in the whole range of masses that we have considered. Moreover, for $T \sim m_{\pi}$ the large number of pions present will further contribute to the thermalization of the heavy hadrons, and for the charged ones also the scattering off photons and electrons will contribute, leading in general to a bound higher than (11).

Now, in order to see what kind of superheavy elements we should expect to find at the present time, and where we should look for them, we will follow the evolution of the heavy quarks from the confinement transition until now.

From $T_{c}$ and up to $T \sim 1 \mathrm{MeV}$, the electroweak interactions among the different heavy hadrons (for instance $\bar{Q} d+\nu \leftrightarrow \bar{Q} u+e$ ) will determine, due to the mass difference of some MeV's between the up and down quarks, an excess of $\bar{Q} u$ over $\bar{Q} d$. Their abundances should have the ration

$$
\frac{n_{i}}{n_{j}}=e^{-\left(m_{i}-m_{j}\right) / T}
$$

and we expect their mass difference to be comparable to the ordinary kaon or B-meson mass splitting.

For the heavy baryon, we will assume that the neutral isosinglet particle state $(Q u d)_{I=0}^{I_{3}=0}$ is lighter than the positively charged member of the isotriplet $(Q u u)_{I=1}^{I_{3}=1}$. This assumption is based on the same kind of analysis that explains qualitatively the mass relationship $m_{\Lambda^{0}}<m_{\Sigma^{+}}$: for $s$-wave baryons, the antisymmetry of the isosinglet $(u d)_{A}$ state in the internal isotopic-spin space forces the spins of the two light quarks to be antiparallel, and the energy of this configuration is lower with respect to the energy of the symmetric $(u d)_{S}$ triplet diquark state, that implies aligned spins [9].

At $T \sim 1 \mathrm{MeV}$, electroweak interactions freeze out and primordial nucleosynthesis has begun. At these temperatures we expect that most of the heavy mesons will be positively charged $\bar{Q} u$, while most of the heavy baryons should be $Q(u d)_{A}$ neutral isosinglets. The surviving neutral $\bar{Q} d$ mesons, if they do not bind to any nucleus, will weakly decay with a typical meanlife of a few seconds. If they bind to nuclei, their decay rate depends on the Coulomb barrier that they feel inside the positively charged nuclei, but in all cases they finish as charged elements. In contrast, due to the large mass splitting $(\mathrm{O}(100 \mathrm{MeV}))$, the few surviving isotriplet baryons should decay electroweakly into the neutral isosinglet even if they bind to nucleons. During nucleosynthesis, a fraction of these neutral baryons may bind to protons (and neutrons) giving rise to superheavy positively charged elements.

This fact has an important consequence, because when galaxies form, after the so called 'violent relaxation', all the atoms that have fallen into the potential well of the galaxy become ionized and, as shown in ref. [10], the charged elements lighter than $\sim 20$ 
$\mathrm{TeV}$ fall into the disk together with the ordinary baryons. So for $m \lesssim 20 \mathrm{TeV}$ we expect most of the charged heavy elements to be found in the disk, while for larger masses they should originally remain mainly in the halos of galaxies. However, larger concentrations of superheavy elements in the disk are to be expected also for masses $>20 \mathrm{TeV}$, since these particles can be captured by the disk during the following evolution of the galaxy. It was recently suggested [11] that this could happen efficiently for particle masses up to $10^{5} \mathrm{TeV}$. Clearly the heavy baryons that do not form charged nuclei during nucleosynthesis will for the most part remain in the halo, leading to an asymmetry between $Q$ and $\bar{Q}$ concentrations in the disk. The heavy elements that had fallen into the disk will be subject to stellar nucleosynthesis forming also superheavy nuclei of large $Z$.

For $m \lesssim 20 \mathrm{TeV}$ the proportion of heavy hadrons $H$ with respect to ordinary nucleons present in the stars (and in the earth) should be of the order of the ratio of their cosmological densities: $n_{H} / n_{b a r}=\left(\Omega_{H} / m\right) /\left(\Omega_{b a r} / m_{p}\right)$, with $m_{p}$ the proton mass. The resulting concentrations are enormously large $\left(\gtrsim 10^{-9}\right)$ and exceed by several orders of magnitude the existing experimental bounds. For instance, searches of superheavy water exclude concentrations of heavy hadrons with respect to ordinary hydrogen in water larger than $\sim 10^{-28}$ for masses $\lesssim 1 \mathrm{TeV}[12]$, larger than $\sim 10^{-24}$ for $m \leq 10 \mathrm{TeV}[13]$, and with less certainty $>10^{-15}$ for larger masses [12]. Actually, in the earth, we expect an even larger concentration of elements containing $H$ hadrons, since they did not evaporate away as most of the ordinary light elements did during the earth lifetime.

For $m>20 \mathrm{TeV}$ the heavy elements remain as dark matter or are captured in the disk, giving a contribution to the density of the galactic halo $\rho_{h}$ in the neighborhood of the disk of at least $\Omega_{h} \cdot \rho_{h}$. However, the existence of large amounts of heavy CHArged Massive Particles, (CHAMPS, ref. [10]) has been recently shown to be in contradiction with the observed long life of several neutron stars [6]. This is due to the fact that CHAMPS captured by the protostellar cloud should collapse into the interior of the stars forming a black hole that would destroy the star in a time scale $\sim$ yr. For the black hole to form it is necessary that the total mass of the captured heavy particles exceeds the Chandrasekhar mass, so that degeneracy does not prevent the gravitational collapse. Since the capture by the protostellar cloud depends on the electromagnetic cross section off hydrogen [6], although the bounds were deduced for leptonic CHAMPS, they also hold for the charged hadronic superheavies under consideration. In the range of masses $20 \mathrm{TeV} \lesssim m \lesssim 10^{5}$ $\mathrm{TeV}$, which is less constrained by searches of superheavy elements, contributions to the halo densities larger than $10^{-7}-10^{-8}$ are ruled out by this argument. (This should be compared with our prediction of more than $10^{-6}$ in this mass range.)

For $E_{6}$ models, since nearly all the $\bar{Q}$ 's form charged elements (e.g. $\left.\bar{Q} u\right)$, while a 
sizeable fraction of the $Q$ 's give rise to neutral states that are not efficiently captured by the protostellar cloud, we expect that a $\bar{Q}-Q$ asymmetry will be present inside the neutron star. This should be a general feature of models for which $Q$ and $\bar{Q}$ belong to hadrons of different charge, or even both neutral, since in these cases we expect that they should bind differently with nuclei [5]. As a consequence, they should be captured by the protostellar cloud at different rates due to their 'chemical' difference, and since even a tiny asymmetry ( $\lesssim 1 \%$ ) between the concentrations of $Q$ and $\bar{Q}$ would leave, after eventual $\bar{Q}-Q$ annihilation inside the star, enough superheavies to produce a black hole, the same conclusions deduced in ref. [6] still hold in our case.

In the case in which $\bar{Q}-Q$ were to form hadrons of equal charge, the large Coulomb barrier will prevent them from annihilating at the typical temperatures of neutron stars.

The previous analysis then leads to the conclusion that the existence of a stable exotic heavy quark can be safely ruled out.

We also note that in the presence of a particle-antiparticle cosmic asymmetry between the heavy quarks, all the bounds would be stronger: the relic abundance would clearly result larger, and hence the maximum cosmologically allowed mass would be smaller. For instance, an asymmetry $n_{Q}-n_{\bar{Q}} / n_{\gamma} \sim 10^{-10}$, which is comparable to the ordinary baryonic one, implies $m \lesssim 250 \mathrm{GeV}$, and the corresponding larger density of relic superheavies would enhance the contradiction with the bounds previously discussed.

Our results have been based on the very accurate determination of the concentration of heavy hadrons in water, and on the observation of long lived neutron stars, but it should be mentioned that several other experimental data (e.g. concentrations of heavy isotopes of different elements, bounds from satellite detectors, etc.) as well as other theoretical considerations (e. $g$. the possible influence of heavy hadrons on stellar evolution) also constrain the exotic quark mass. For instance, the stringent bounds on strongly interacting dark matter that come both from detector searches near the top of the atmosphere [14] as well as from underground experiments [15], will apply to the neutral superheavy baryons, restricting thus their possible contribution to the local density of the halo (the charged component is stopped before it can reach the detectors mentioned).

Our conclusion is that a stable or very long lived quark would be present with too large a density to be compatible with the cosmological requirements, with the bounds obtained from anomalous element searches and with some astrophysical implications. Hence, models with exotic quarks must include also a mechanism to allow for their decay. In the case of $E_{6}$ models this can be achieved by allowing for the presence of nonvanishing couplings of $Q$ with other (scalar) particles that could mediate their decay or induce, through a non zero vacuum expectation value, a sizeable mixing among exotic and ordinary quarks. 


\section{Acknowledgements}

We are grateful to S. Dimopoulos, J. Ellis, G. F. Giudice and N. Tetradis for several useful discussions. 


\section{References}

[1] For a review on the group $E_{6}$, see R.Slansky, Phys.Rep. 79, No.1 (1981) 1.

[2] P.Candelas, G.Horowitz, A.Strominger, and E.Witten,Nucl.Phys. B 258 (1985) 46;

E.Witten, Nucl.Phys. B 258 (1985) 75.

[3] UA1 Collaboration, CERN-EP/89 - 146.

[4] J.L. Hewett and T.G. Rizzo, Phys.Rep. $1835 \& 6$ (1989) 195 and references therein.

[5] C.B. Dover, T.K. Gaisser and G. Steigman; Phys. Rev. Lett. 42 (1979) 1117.

[6] A.Gould,B.Draine,R.Romani and S.Nussinov; IASSNS-AST 89/55.

[7] Lee and Weinberg; PRL 39, 165 (1977)

K. Griest and D. Seckel; Nucl. Phys. B 283 (1987) 621

[8] D.J. Gross, R.D. Pisarsky and L.G. Yaffe, Rev. Mod. Phys. 53 (1981) 43.

[9] A.De Rújula, H.Georgi and S.L.Glashow; Phys. Rev. D12 (1975) 147

[10] A. de Rújula, S. Glashow and U. Sarid; CERN-TH. 5490/89.

[11] S. Dimopoulos, D. Eichler, R. Esmailzadeh, G. Starkman; IASSNS-AST 89/53.

[12] P.F. Smith et. al.; Nucl. Phys. B206 (1982) 333.

[13] T.K. Hemmick et. al. ; Nucl. Phys. B29 (1987) 389.

[14] J.Rich, R.Rocchia and M.Spiro; Phys. Lett. B194 (1987) 173.

[15] S.P. Ahlen et al.; Phys. Rev. D36 (1987) 311.

\section{Figure Captions}

\section{Fig. 1}

Relic density of exotic stable quarks as a function of their mass for annihilation cross sections after confinement of $100 \mathrm{mb}(f=1), 10 \mathrm{mb}(f=0.1)$ or negligible $(f=0)$. 


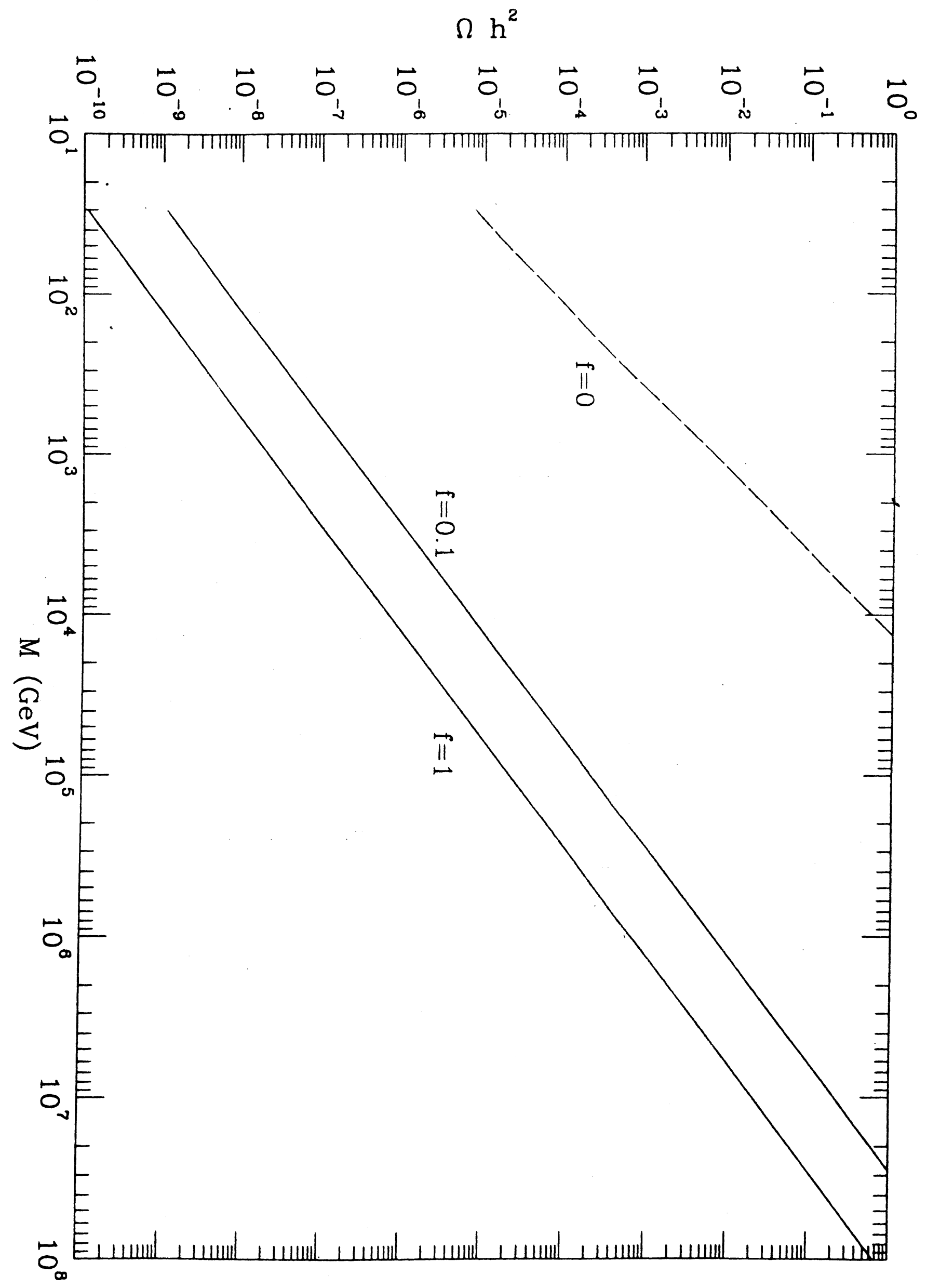

